\title{
Effect of Weed Control Methods on Weeds and Wheat under Eucalyptus tereticornis Based Agroforestry System
}

\author{
Atul Singh", K.K. Jain and S.D. Upadhyaya \\ Department of forestry, JNKVV Jabalpur (MP) pin no. - 482004, India \\ *Corresponding author
}

\begin{tabular}{|l|}
\hline Ke y w o r d s \\
Weeds, Weed \\
control, Grain yield, \\
Straw yield
\end{tabular}

\section{Introduction}

Agroforestry is a sustainable land use system where two or more component is growing simultaneously on the same unit of land. Agroforestry may be one of the solutions to increase area outside the forest to one third of the total geographical area of our country.

The importance of agroforestry land use for food, fuel, fodder, fruits, fertilizer, timber, etc.
A field experiment was conducted during winter season to find out the effect of herbicides on weed dynamics and yield performance of wheat (Triticum aestivum L.) variety LOK-1 in randomized complete block design with three replications under eucalyptus based agroforestry system. An experiment was conducted at the farmer field village- Majitha, District- Jabalpur during the rabi season of 2016-17 and 2017-18. The field was infested with 5 major weed species Phalaris minor, Rumex dentatus (L.), Melilotus indicus (L.), Chenopodium album (L.) and Launaea nudicaulis (L.) during both the year. The hand weeding showed minimum total weed density and dry weight and proved more effective than all weed control treatments and over weedy check. Among chemical weed control treatment 2, 4-D 0.5 lit ha $^{-1}+$ hand weeding 30 DAS and 2, 4-D 0.5 lit ha $^{-1} \mathrm{fb}$ metribuzin $0.250 \mathrm{Kg} \mathrm{ha}^{-1}$, Metribuzin $0.250 \mathrm{Kg} \mathrm{ha}^{-1}$ have control both broad leaf and grassy weed and Clodinafop-propargyl $0.140 \mathrm{~kg} \mathrm{ha}^{-1}$ control grassy weed over weedy check. The application of 2, 4-D 0.5 lit ha $^{-1} \mathrm{fb}$ metribuzin $0.250 \mathrm{Kg} \mathrm{ha}^{-1}$ (T5: 77.03 and 80.41\%), 2, 4D 0.5 lit ha $^{-1}$ (T1: 75.15 and 83.00\%), and 2, 4-D 0.5 lit ha $^{-1}+$ hand weeding 30 DAS (T8: 75.18 and $77.60 \%$ ) was found higher weed control efficiency during both the year under wheat- Eucalyptus tereticornis based agroforestry system. The hand weeding showed maximum weed control efficiency (T9: 86.73 and 95.51\%) and proved superior over herbicidal treatments. The higher grain yield and straw yield was found under hand weeding 30 DAS (T9; 19.75, 18.20 and 46.54, $39.72 \mathrm{q} \mathrm{ha}^{-1}$ ) during both the year. 
reducing unemployment, generating additional source of income and livelihood support to small landholders (Verma et al., 2017).

Wheat (Triticum aestivum L.) is the major cereal crop in many dry areas of the world and a basic food for more than one third of the world population. It is a prime source of carbohydrates and protein which has served as a staple diet for mankind (Nural-lslam and Johanson, 1987). Ecologically, wheat is adapted to a variety of climates and stressed environments including salinity. However, different biotic and abiotic stresses cause reduction in grain yield to various extents depending upon their nature and intensity. In agroforestry systems, reduction in yield of wheat is generally observed under the shade of tree crown and weeds due to resource competition (Puri and Bangarwa, 1992 and Awan et al., 2015).

Weed infestation is one of the major factor limiting crop productivity. For realizing full genetic yield potential of the crop, the proper weed control is one of the essential management practices. Weeds not only reduce the yield but also make the harvesting operation difficult. Therefore, for sustaining food grain production to feed ever-increasing population and ensuring food security, effective weed management is very essential. Uncontrolled weeds are reported to cause upto $66 \%$ reduction in wheat grain yield (Angiras et al., 2008, Kumar et al., 2010 and Kumar et al., 2011) or even more depending upon the weed density, type of weed flora and duration of infestation. In wheat growing bowl of the country, infestation of grassy weeds likes $P$. minor and Avena ludoviciana L. and broadleaf weeds like Chenopodium album L., Chichorium intybus L. and Rumex dentates L. etc are increasing at an alarming rate thus culminating wheat yield reduction by 18 to $73 \%$. To manage the dynamics of weed flora, there is a need to evaluate a range of herbicides to have broad spectrum weed control. Chemical weed control is a preferred practice due to scarce, costly labour and time consuming as well as lesser feasibility of mechanical or manual weeding especially in broadcast wheat (Dixit and Singh, 2008). Hence, an experiment was conducted to evaluate the effect of weed control treatments, herbicides and their mixtures on weeds and wheat yield under Eucalyptus tereticornis based agroforestry system.

\section{Materials and Methods}

The field experiment was conducted at farmer's field during Rabi season 2016 -17 and 2017-18 at Village - Majitha, Block Shahpura, District - Jabalpur. The experiment was laid out in randomized block design with three replications and consisted of ten weed control treatment $\left[2,4-\mathrm{D} \quad 0.5\right.$ lit $\mathrm{ha}^{-1}$, Metribuzin $0.250 \mathrm{Kg} \mathrm{ha}^{-1}$, Butachlor 1 lit ha $^{-1}$, Clodinafop-propargyl $0.140 \mathrm{~kg} \mathrm{ha}^{-1}, 2$, 4-D 0.5 lit ha ${ }^{-1} \mathrm{fb}$ metribuzin $0.250 \mathrm{Kg} \mathrm{ha}^{-1}, 2$, 4-D 0.5 lit ha ${ }^{-1} \mathrm{fb}$ Butachlor 1 lit ha ${ }^{-1}$, Metribuzin 0.250 $\mathrm{Kg} \mathrm{ha}^{-1} \mathrm{fb}$ butachlor 1 lit ha ${ }^{-1}, 2$, 4-D 0.5 lit ha 1 + hand weeding 30 DAS, Hand weeding 30 DAS and Weedy check]. Wheat variety LOK1 was sown with $25 \mathrm{~cm}$ row spacing at a depth of $4 \mathrm{~cm}$ from the top of the soil by opening furrows through a Kudal. The weed control treatments and herbicides were applied as post emergent at crop tillering stage i.e. about 30 DAS. Weed population was counted with the help of quadrate $(0.25 \mathrm{~cm} X 0.25 \mathrm{~cm})$ thrown randomly at four places in each plot and converted in to $\mathrm{m}^{2}$ area. The aboveground weed dry matter was also recorded from the above thrown quadrates after cutting weeds from the ground level and then oven dried at $70^{\circ} \mathrm{C}$ and converted to $\mathrm{m}^{2}$. The yield of crop was recorded in all the treatments at the time of harvest. Harvest index was calculated as the ratio of grain yield to the biological yield. It was calculated as per the formula proposed by Nichiporovich (1967). 
Harvest Index = $-\frac{\text { Economic yield }}{\text { Biological yield }}$

\section{Weed control efficiency (WCE)}

Weed control efficiency (WCE) of the treatments against weedy check was calculated on the basis of weed dry weight as suggested by Mani et al., (1973).

$\mathrm{WCE}(\%)=\frac{\mathrm{WD}_{\mathrm{c}}-\mathrm{WD}_{\mathrm{t}}}{\mathrm{WD}_{\mathrm{c}}}$

Where,

WCE $=$ Weed control efficiency

$\mathrm{WD}_{\mathrm{c}}=$ Dry weight of weeds in unweeded control plot

$\mathrm{WD}_{\mathrm{t}}=$ Dry weight of weeds in treated plot

Weed count were subjected to square root transformation, $(\sqrt{\mathrm{X}}+0.5)$.

\section{Weed index}

Weed index of each treatment was calculated by using following formula (Gill and Kumar, 1969).

Weed Index $(\%)=\frac{\mathrm{X}-\mathrm{Y}}{\mathrm{-} \text { X }}$

Where,

$X$ - Yield from hand weeded plot.

$\mathrm{Y}$ - Yield from the treatment for which weed index is to be worked out.

Weed count were subjected to square root transformation, $\sqrt{X}+0.5$. All the experimental data were statistically analyzed and critical difference (CD) was worked out by the procedure as described by Gomez and Gomez (1984).

\section{Results and Discussion}

\section{Weed flora}

The weed community comprised both broadleaved and grass weeds. The experiment field consisted with 5 weed species belonging to 5 families in the experimental plot.

\section{Effect on individual weeds}

\section{Phalaris minor}

The perusal of data showed that hand weeding found lower weed density (T9: 2.00 and 0.67 $\mathrm{m}^{2}$ ) over all the weed control treatment and weedy check during both the year. Among chemical weed control treatment Clodinafoppropargyl $0.140 \mathrm{~kg} \mathrm{ha}^{-1}$ showed lowest weed density (T4: 2.00 and $1.00 \mathrm{~m}^{2}$ ) followed by Metribuzin $0.250 \mathrm{Kg} \mathrm{ha}^{-1}$ (T2: 3.33 and 2.00 $\mathrm{m}^{2}$ ) 2, 4-D 0.5 lit ha $^{-1} \mathrm{fb}$ metribuzin $0.250 \mathrm{Kg}$ $\mathrm{ha}^{-1}$ (T5: 3.33 and $2.67 \mathrm{~m}^{2}$ ) and 2, 4-D 0.5 lit $\mathrm{ha}^{-1}+$ hand weeding 30 DAS (T8: 3.673 .50 $\mathrm{m}^{2}$ ) over weedy check which recorded significantly higher weed control treatment (T10: 9.33 and $11.33 \mathrm{~m}^{2}$ ) during both the year under wheat - Eucalyptus tereticornis based Agroforestry system (Table 1). The perusal of statistical data reported that highest weed control efficiency was found under hand weeding 30DAS (T9: 77.50 and 94.10\%) followed by clodinafop-propargyl $0.140 \mathrm{~kg}$ ha $^{-1}$ (T4: 77.50 and 91.84 \%) over weedy check (T10- $0 \%), 2$, 4-D 0.5 lit ha $^{-1}$ (T1:13.33 and $52.03 \%$ ) and butachlor 1 lit ha ${ }^{-1}$ (T3: 32.50 and $58.70 \%$ ) during both the year (Table 2) Similar views have also been reported by, Singh et al., (2005), Amare et al., (2014), Saini and Chopra (2015) and Singh et al., (2015). 
The perusal of data (Table 3) showed that the lowest dry weight of Phalaris minor was recorded in hand weeding 30 DAS (T9: 4.00 and $1.33 \mathrm{gm}^{-2}$ ) at par with clodinafoppropargyl $0.140 \mathrm{~kg} \mathrm{ha}^{-1}$ (T4: 4.00 and 2.00 $\left.\mathrm{gm}^{-2}\right)$. The weedy check recorded higher weed dry weight (T10: 18.67 and $22.67 \mathrm{gm}^{-2}$ ) during both year. Similar finding was also reported by Pradhan and Chakraborti (2010), Tiwari et al., (2011), Pisal and Sagarka (2013), Amare et al., (2014) and choudhry et al., (2016).

\section{Chenopodium album}

The result showed that hand weeding recorded lower weed density (T9: 1.33 and $0.67 \mathrm{~m}^{2}$ ) whereas weedy check recorded significantly higher weed density (T10: 9.67 and $8.67 \mathrm{~m}^{2}$ ). Among chemical weed control treatment 2, 4D 0.5 lit ha ${ }^{-1}$ showed lower weed density (T1: 1.33 and $1.00 \mathrm{~m}^{2}$ ) followed by 2, 4-D 0.5 lit $\mathrm{ha}^{-1}+$ hand weeding 30 DAS (T8: 2.00 and $2.00 \mathrm{~m}^{2}$ ) and 2, 4-D 0.5 lit ha $^{-1} \mathrm{fb}$ metribuzin $0.250 \mathrm{Kg} \mathrm{ha}^{-1}$ (T5: 2.33 and $2.5 \mathrm{~m}^{2}$ ) during both the year under wheat - Eucalyptus tereticornis based Agroforestry system (Table $1)$.

The application of weed management practices the highest weed control efficiency was found under hand weeding 30 DAS (T9:
86.11 and $93.94 \%)$. The application of 2, 4-D 0.5 lit ha $^{-1}$ (T1: 84.26 and $87.45 \%$ ), 2, 4-D 0.5 lit $\mathrm{ha}^{-1}+$ hand weeding 30 DAS (T8: 79.17 and $79.74 \%), 2$, 4-D 0.5 lit ha ${ }^{-1} \mathrm{fb}$ metribuzin $0.250 \mathrm{Kg} \mathrm{ha}^{-1}$ (T5: 74.54 and $76.34 \%$ ), metribuzin $0.250 \mathrm{Kg} \mathrm{ha}^{-1}$ (T2: 65.28 and 66.23 $\%)$ recorded higher weed control efficiency over the weedy check (T10: $0 \%$ ) during both the year(Table 2) Similar views have also been reported by, Singh et al., (2005), Amare et al., (2014), Saini and Chopra (2015) and Singh et al., (2015).

The minimum dry weight of Chenopodium album was recorded in hand weeding 30 DAS (T9: 4.00 and $2.00 \mathrm{~g} \mathrm{~m}^{-2}$ ) which was significantly superior over weedy check which recorded higher dry weight (T10: 29.00 and $26.00 \mathrm{~g} \mathrm{~m}^{-2}$ ).

The application of 2, 4-D 0.5 lit ha ${ }^{-1}$ (T1: 4.00 and $3.00 \mathrm{~g} \mathrm{~m}^{-2}$ ), 2, 4-D 0.5 lit ha ${ }^{-1}+$ hand weeding 30 DAS (T8: 6.00 and $4.67 \mathrm{~g} \mathrm{~m}^{-2}$ ) and 2, 4-D 0.5 lit ha ${ }^{-1} \mathrm{fb}$ metribuzin $0.250 \mathrm{Kg}$ $\mathrm{ha}^{-1}$ (T5: 7.00 and $5.67 \mathrm{~g} \mathrm{~m}^{-2}$ ) were also at par with hand weeding which rerecorded minimum dry weight of Chenopodium album during both the year(Table 3). Similar find also reported by Tiwari et al., (2011), Pisal and Sagarka (2013), Amare et al., (2014) and choudhry et al., (2016).

Table.1 Floristic composition of weeds of the experimental field

\begin{tabular}{|c|c|c|c|}
\hline Botanical name & Common name & Family & Habit and characteristics \\
\hline \multicolumn{4}{|l|}{ Grasses } \\
\hline Phalaris minor & Canary grass & Poaceae & $\begin{array}{l}\text { Tufted annual bunch grass, spike } \\
\text { like panicle. }\end{array}$ \\
\hline \multicolumn{4}{|l|}{ Broad leaved weeds } \\
\hline Rumex dentatus (L.) & Toothed dock, Aegean dock & Polygonaceae & Annual, erect with long taproots. \\
\hline Meliotus indica (L.) All. & $\begin{array}{c}\text { Sweet clover, Indian sweet } \\
\text { clover }\end{array}$ & Fabaceae & $\begin{array}{c}\text { Annual herb of } 10-50 \mathrm{~cm} \text {, yellow } \\
\text { flowers. }\end{array}$ \\
\hline Chenopodium album (L.) & Lambsquarters, goosefoot & Chenopodiaceae & $\begin{array}{c}\text { Annual, many branches, dull green } \\
\text { flowers. }\end{array}$ \\
\hline Launaea nudicaulis (L.) & Broad leaf launaea & Asteraceae & $\begin{array}{l}\text { Perennial herb with a taproot and } \\
\text { often shoot bearing lateral roots, up } \\
\text { to } 40-50 \mathrm{~cm} \text { high. }\end{array}$ \\
\hline
\end{tabular}


Table.2 Effect of weed control treatment on weed density $\left(\mathrm{m}^{-2}\right)$ at harvest during both the year under wheat- Eucalyptus tereticornis based agroforestry system

\begin{tabular}{|c|c|c|c|c|c|c|c|c|c|c|}
\hline \multirow[t]{2}{*}{ Treatments } & \multicolumn{2}{|c|}{ Phalaris minor } & \multicolumn{2}{|c|}{$\begin{array}{l}\text { Chenopodium } \\
\text { album }\end{array}$} & \multicolumn{2}{|c|}{ Rumex dentatus } & \multicolumn{2}{|c|}{$\begin{array}{l}\text { Launaea } \\
\text { nudicaulis }\end{array}$} & \multicolumn{2}{|c|}{ Melilotus indicus } \\
\hline & 2016-17 & 2017-18 & 2016-17 & 2017-18 & 2016-17 & 2017-18 & 2016-17 & 2017-18 & 2016-17 & 2017-18 \\
\hline $\mathrm{T}_{1}-2,4-\mathrm{D} 0.5 \mathrm{lit} \mathrm{ha}^{-1}$ & $\begin{array}{c}2.91 \\
(8.00)^{*}\end{array}$ & $\begin{array}{c}2.42 \\
(5.33)\end{array}$ & $\begin{array}{c}1.29 \\
(1.33)\end{array}$ & $\begin{array}{c}1.17 \\
(1.00)\end{array}$ & $\begin{array}{c}1.29 \\
(1.33)\end{array}$ & $\begin{array}{c}1.00 \\
(0.67)\end{array}$ & $\begin{array}{c}1.46 \\
(1.67)\end{array}$ & $\begin{array}{c}1.34 \\
(1.00)\end{array}$ & $\begin{array}{c}1.47 \\
(2.00)\end{array}$ & $\begin{array}{c}1.34 \\
(1.33)\end{array}$ \\
\hline $\begin{array}{l}\mathrm{T}_{2}-\text { Metribuzin } 0.250 \\
\mathrm{Kg} \mathrm{ha}^{-1}\end{array}$ & $\begin{array}{c}1.95 \\
(3.33)\end{array}$ & $\begin{array}{c}1.56 \\
(2.00)\end{array}$ & $\begin{array}{c}1.95 \\
(3.33)\end{array}$ & $\begin{array}{c}1.81 \\
(2.83)\end{array}$ & $\begin{array}{c}1.77 \\
(2.67)\end{array}$ & $\begin{array}{c}1.68 \\
(2.33)\end{array}$ & $\begin{array}{c}1.77 \\
(2.67)\end{array}$ & $\begin{array}{l}1.47 \\
(1.83)\end{array}$ & $\begin{array}{l}1.77 \\
(2.67)\end{array}$ & $\begin{array}{l}1.46 \\
(1.67)\end{array}$ \\
\hline $\mathrm{T}_{3^{-}}-$Butachlor 1 lit ha & $\begin{array}{c}2.61 \\
(6.33)\end{array}$ & $\begin{array}{c}2.27 \\
(4.67)\end{array}$ & $\begin{array}{c}2.20 \\
(4.33)\end{array}$ & $\begin{array}{c}1.95 \\
(3.33)\end{array}$ & $\begin{array}{l}1.76 \\
(2.67)\end{array}$ & $\begin{array}{c}1.73 \\
(2.50)\end{array}$ & $\begin{array}{c}2.11 \\
(4.00)\end{array}$ & $\begin{array}{c}1.94 \\
(3.33)\end{array}$ & $\begin{array}{c}1.90 \\
(3.17)\end{array}$ & $\begin{array}{l}1.77 \\
(2.67)\end{array}$ \\
\hline $\begin{array}{l}\text { T}_{4^{-}} \text {Clodinafop- } \\
\text { propargyl } 0.140 \mathrm{~kg} \mathrm{ha}^{-1}\end{array}$ & $\begin{array}{l}1.48 \\
(2.00)\end{array}$ & $\begin{array}{c}1.17 \\
(1.00)\end{array}$ & $\begin{array}{c}2.48 \\
(5.67)\end{array}$ & $\begin{array}{c}2.34 \\
(5.00)\end{array}$ & $\begin{array}{c}2.26 \\
(4.67)\end{array}$ & $\begin{array}{c}2.00 \\
(3.50)\end{array}$ & $\begin{array}{c}2.04 \\
(3.67)\end{array}$ & $\begin{array}{l}1.78 \\
(2.83)\end{array}$ & $\begin{array}{c}1.86 \\
(3.00)\end{array}$ & $\begin{array}{l}1.68 \\
(2.33)\end{array}$ \\
\hline $\begin{array}{l}\mathrm{T}_{5^{-}} 2,4-\mathrm{D} 0.5 \mathrm{lit} \mathrm{ha}^{-1} \mathrm{fb} \\
\text { metribuzin } 0.250 \mathrm{Kg} \\
\mathrm{ha}^{-1}\end{array}$ & $\begin{array}{l}1.95 \\
(3.33)\end{array}$ & $\begin{array}{c}1.77 \\
(2.67)\end{array}$ & $\begin{array}{c}1.68 \\
(2.33)\end{array}$ & $\begin{array}{l}1.72 \\
(2.5)\end{array}$ & $\begin{array}{l}1.46 \\
(1.67)\end{array}$ & $\begin{array}{l}1.46 \\
(1.6)\end{array}$ & $\begin{array}{c}1.39 \\
(1.67)\end{array}$ & $\begin{array}{l}1.29 \\
(1.33)\end{array}$ & $\begin{array}{c}1.29 \\
(1.33)\end{array}$ & $\begin{array}{c}1.34 \\
(1.33)\end{array}$ \\
\hline $\begin{array}{l}\mathrm{T}_{6-}, 2,4-\mathrm{D} 0.5 \mathrm{lit} \mathrm{ha}^{-1} \mathrm{fb} \\
\text { butachlor } 1 \mathrm{lit} \mathrm{ha}^{-1} \\
\end{array}$ & $\begin{array}{c}2.27 \\
(4.67)\end{array}$ & $\begin{array}{c}2.11 \\
(4.00)\end{array}$ & $\begin{array}{c}2.11 \\
(4.00)\end{array}$ & $\begin{array}{c}1.91 \\
(3.17)\end{array}$ & $\begin{array}{l}1.77 \\
2.67\end{array}$ & $\begin{array}{c}1.63 \\
(2.17)\end{array}$ & $\begin{array}{c}1.86 \\
(3.00)\end{array}$ & $\begin{array}{l}1.74 \\
(2.60)\end{array}$ & $\begin{array}{l}1.76 \\
(2.67)\end{array}$ & $\begin{array}{l}1.66 \\
(2.27)\end{array}$ \\
\hline $\begin{array}{l}\mathrm{T}_{8^{-}} \text {2, 4-D } 0.5 \text { lit ha } \\
\text { hand weeding } 30 \text { DAS }\end{array}$ & $\begin{array}{c}2.03 \\
(3.67)\end{array}$ & $\begin{array}{c}1.98 \\
(3.50)\end{array}$ & $\begin{array}{c}1.48 \\
(2.00)\end{array}$ & $\begin{array}{c}1.56 \\
(2.00)\end{array}$ & $\begin{array}{c}1.56 \\
(2.00)\end{array}$ & $\begin{array}{c}1.29 \\
(1.33)\end{array}$ & $\begin{array}{c}1.56 \\
(2.00)\end{array}$ & $\begin{array}{l}1.48 \\
(2.00)\end{array}$ & $\begin{array}{l}1.68 \\
(2.33)\end{array}$ & $\begin{array}{l}1.68 \\
(2.33)\end{array}$ \\
\hline $\begin{array}{l}\text { T9- Hand Weeding } 30 \\
\text { DAS }\end{array}$ & $\begin{array}{l}1.48 \\
(2.00)\end{array}$ & $\begin{array}{c}1.05 \\
(0.67)\end{array}$ & $\begin{array}{c}1.27 \\
(1.33)\end{array}$ & $\begin{array}{c}1.00 \\
(0.67)\end{array}$ & $\begin{array}{c}1.05 \\
(0.67)\end{array}$ & $\begin{array}{c}0.71 \\
(0.00)\end{array}$ & $\begin{array}{c}1.17 \\
(1.00)\end{array}$ & $\begin{array}{c}0.88 \\
(0.33)\end{array}$ & $\begin{array}{c}1.00 \\
(0.67)\end{array}$ & $\begin{array}{c}1.05 \\
(0.67)\end{array}$ \\
\hline $\mathrm{T}_{10^{-}}$Weedy check & $\begin{array}{c}3.13 \\
(9.33)\end{array}$ & $\begin{array}{c}3.43 \\
(11.33)\end{array}$ & $\begin{array}{c}3.18 \\
(9.67)\end{array}$ & $\begin{array}{c}3.02 \\
(8.67)\end{array}$ & $\begin{array}{c}2.80 \\
(7.33)\end{array}$ & $\begin{array}{c}2.74 \\
(7.00)\end{array}$ & $\begin{array}{c}2.97 \\
(8.33)\end{array}$ & $\begin{array}{c}2.80 \\
(8.33)\end{array}$ & $\begin{array}{l}3.01 \\
(8.67)\end{array}$ & $\begin{array}{c}3.13 \\
(9.33)\end{array}$ \\
\hline SEm \pm & 0.22 & 0.14 & 0.22 & 0.19 & 0.18 & 0.18 & 0.18 & 0.16 & 0.21 & 0.11 \\
\hline $\begin{array}{l}\text { Treatment (T) CD } \\
(P=0.05)\end{array}$ & 0.66 & 0.42 & 0.64 & 0.55 & 0.53 & 0.51 & 0.54 & 0.47 & 0.62 & 0.33 \\
\hline
\end{tabular}

$*$ (Data subjected to square root $\mathrm{x}+0.5$ transformation and figures in parenthesis are original value) 
Table.3 Effect of weed control treatment on weed control efficiency (\%) under wheatEucalyptus tereticornis based agroforestry system

\begin{tabular}{|c|c|c|c|c|c|c|c|c|c|c|}
\hline \multirow[t]{2}{*}{ Treatments } & \multicolumn{2}{|c|}{ Phalaris minor } & \multicolumn{2}{|c|}{$\begin{array}{c}\text { Chenopodium } \\
\text { album }\end{array}$} & \multicolumn{2}{|c|}{ Rumex dentatus } & \multicolumn{2}{|c|}{$\begin{array}{l}\text { Launaea } \\
\text { nudicaulis }\end{array}$} & \multicolumn{2}{|c|}{ Melilotus indicus } \\
\hline & 2016-17 & 2017-18 & 2016-17 & 2017-18 & 2016-17 & & 2016-17 & 2017-18 & 2016-17 & 2017-18 \\
\hline $\mathrm{T}_{1}-2,4-\mathrm{D} 0.5 \mathrm{lit} \mathrm{ha}^{-1}$ & 13.33 & 52.03 & 84.26 & 87.45 & 84.26 & 91.67 & 79.63 & 86.90 & 79.37 & 81.39 \\
\hline $\begin{array}{l}\mathrm{T}_{2}-\text { Metribuzin } 0.250 \\
\mathrm{Kg} \mathrm{ha}^{-1}\end{array}$ & 64.17 & 82.45 & 65.28 & 66.23 & 65.28 & 66.17 & 67.59 & 77.86 & 67.86 & 75.00 \\
\hline $\mathrm{T}_{3}-$ Butachlor 1 lit ha ${ }^{-1}$ & 32.50 & 58.70 & 53.70 & 59.96 & 53.70 & 63.10 & 51.85 & 59.29 & 61.71 & 71.94 \\
\hline $\begin{array}{l}\mathrm{T}_{4-} \text { Clodinafop- } \\
\text { propargyl } 0.140 \mathrm{~kg} \\
\mathrm{ha}^{-1}\end{array}$ & 77.50 & 91.84 & 38.42 & 41.94 & 38.42 & 50.00 & 56.02 & 65.60 & 65.08 & 69.72 \\
\hline $\begin{array}{l}\mathrm{T}_{5^{-}} \text {2, 4-D } 0.5 \mathrm{lit}^{\mathrm{h} \mathrm{A}^{-1} \mathrm{fb}} \\
\text { metribuzin } 0.250 \mathrm{Kg} \\
\mathrm{ha}^{-1}\end{array}$ & 63.33 & 75.78 & 74.54 & 76.34 & 74.54 & 76.59 & 80.09 & 82.14 & 80.95 & 86.94 \\
\hline 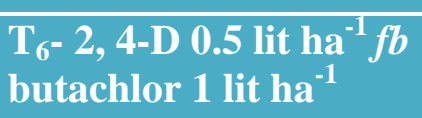 & 49.17 & 64.59 & 58.33 & 63.48 & 58.33 & 68.65 & 64.35 & 68.33 & 69.84 & 76.53 \\
\hline $\begin{array}{l}\mathrm{T}_{7} \text { - Metribuzin } 0.250 \\
\mathrm{Kg} \mathrm{ha}^{-1} \mathrm{fb} \text { butachlor } 1 \\
\text { lit ha }^{-1}\end{array}$ & 50.83 & 67.24 & 39.81 & 46.16 & 39.81 & 49.01 & 68.06 & 72.14 & 65.08 & 68.33 \\
\hline $\begin{array}{l}\mathrm{T}_{8-} \text { 2, 4-D } 0.5 \text { lit ha }{ }^{-1}+ \\
\text { hand weeding } 30 \text { DAS }\end{array}$ & 60.83 & 68.44 & 79.17 & 79.74 & 79.17 & 80.56 & 75.46 & 75.71 & 70.63 & 76.11 \\
\hline $\begin{array}{l}\text { T9- Hand Weeding } 30 \\
\text { DAS }\end{array}$ & 77.50 & 94.10 & 86.11 & 93.94 & 86.11 & 100.00 & 87.96 & 95.83 & 94.44 & 93.06 \\
\hline $\mathrm{T}_{10^{-}}$Weedy check & 0.00 & 0.00 & 0.00 & 0.00 & 0.00 & 0.00 & 0.00 & 0.00 & 0.00 & 0.00 \\
\hline SEm \pm & 7.68 & 4.30 & 7.03 & 4.94 & 7.03 & 6.07 & 6.45 & 6.28 & 6.68 & 4.26 \\
\hline $\begin{array}{l}\text { Treatment (T) CD } \\
(P=0.05)\end{array}$ & 22.42 & 12.54 & 20.52 & 14.41 & 20.52 & 17.72 & 18.82 & 18.33 & 19.50 & 12.43 \\
\hline
\end{tabular}


Table.4 Effect of weed control treatment on weed dry weight $\left(\mathrm{g} \mathrm{m}^{-2}\right)$ under wheat - Eucalyptus tereticornis based agroforestry system

\begin{tabular}{|c|c|c|c|c|c|c|c|c|c|c|}
\hline \multirow[t]{2}{*}{ Treatments } & \multicolumn{2}{|c|}{ Phalaris minor } & \multicolumn{2}{|c|}{$\begin{array}{l}\text { Chenopodium } \\
\text { album }\end{array}$} & \multicolumn{2}{|c|}{ Rumex dentatus } & \multicolumn{2}{|c|}{ Launaea nudicaulis } & \multicolumn{2}{|c|}{ Melilotus indicus } \\
\hline & 2016-17 & 2017-18 & 2016-17 & 2017-18 & 2016-17 & 2016-17 & 2017-18 & 2016-17 & 2017-18 & 2016-17 \\
\hline $\mathrm{T}_{1}-2,4-\mathrm{D} 0.5 \mathrm{lit} \mathrm{ha}^{-1}$ & $\begin{array}{c}4.06 \\
(16.00)\end{array}$ & $\begin{array}{c}3.34 \\
(10.67)\end{array}$ & $\begin{array}{c}1.90 \\
(4.00)\end{array}$ & $\begin{array}{c}1.71 \\
(3.00)\end{array}$ & $\begin{array}{c}2.57 \\
(8.00)\end{array}$ & $\begin{array}{c}1.65 \\
(4.00)\end{array}$ & $\begin{array}{c}3.17 \\
(10.00)\end{array}$ & $\begin{array}{c}2.27 \\
(6.00)\end{array}$ & $\begin{array}{c}2.23 \\
(6.00)\end{array}$ & $\begin{array}{c}2.27 \\
(5.00)\end{array}$ \\
\hline $\begin{array}{l}\mathrm{T}_{2} \text { - Metribuzin } 0.250 \\
\mathrm{Kg} \mathrm{ha}^{-1}\end{array}$ & $\begin{array}{c}2.67 \\
(6.67)\end{array}$ & $\begin{array}{c}2.08 \\
(4.00)\end{array}$ & $\begin{array}{c}3.23 \\
(10.00)\end{array}$ & $\begin{array}{c}2.97 \\
(8.50)\end{array}$ & $\begin{array}{c}4.50 \\
(20.00)\end{array}$ & $\begin{array}{c}3.81 \\
(14.00)\end{array}$ & $\begin{array}{c}4.03 \\
(16.00)\end{array}$ & $\begin{array}{c}3.39 \\
(11.00)\end{array}$ & $\begin{array}{c}2.90 \\
(8.00)\end{array}$ & $\begin{array}{c}2.82 \\
(7.50)\end{array}$ \\
\hline $\mathrm{T}_{3^{-}}$Butachlor 1 lit ha ${ }^{-1}$ & $\begin{array}{c}3.61 \\
(12.67)\end{array}$ & $\begin{array}{c}3.13 \\
(9.33)\end{array}$ & $\begin{array}{c}3.63 \\
(13.00)\end{array}$ & $\begin{array}{c}3.23 \\
(10.00)\end{array}$ & $\begin{array}{c}5.10 \\
(26.00)\end{array}$ & $\begin{array}{c}3.93 \\
(15.00)\end{array}$ & $\begin{array}{c}4.90 \\
(24.00)\end{array}$ & $\begin{array}{c}4.48 \\
(20.00)\end{array}$ & $\begin{array}{c}3.10 \\
(9.50)\end{array}$ & $\begin{array}{c}2.90 \\
(8.00)\end{array}$ \\
\hline $\begin{array}{l}\text { T4- Clodinafop- } \\
\text { propargyl } 0.140 \mathrm{~kg} \mathrm{ha}^{-1}\end{array}$ & $\begin{array}{c}1.94 \\
(4.00)\end{array}$ & $\begin{array}{c}1.47 \\
(2.00)\end{array}$ & $\begin{array}{c}4.13 \\
(17.00)\end{array}$ & $\begin{array}{c}3.93 \\
(15.00)\end{array}$ & $\begin{array}{c}5.83 \\
(34.00)\end{array}$ & $\begin{array}{c}4.63 \\
(21.00)\end{array}$ & $\begin{array}{c}4.70 \\
(22.00)\end{array}$ & $\begin{array}{c}4.18 \\
(17.00)\end{array}$ & $\begin{array}{c}3.03 \\
(9.00)\end{array}$ & $\begin{array}{c}3.06 \\
(9.00)\end{array}$ \\
\hline $\begin{array}{l}\mathrm{T}_{5^{-}} 2,4-\mathrm{D} 0.5 \mathrm{lit}^{\mathrm{ha}} \mathrm{fb}^{-1} \\
\text { metribuzin } 0.250 \mathrm{Kg} \\
\mathrm{ha}^{-1}\end{array}$ & $\begin{array}{c}2.67 \\
(6.67)\end{array}$ & $\begin{array}{c}2.41 \\
(5.33)\end{array}$ & $\begin{array}{c}2.70 \\
(7.00)\end{array}$ & $\begin{array}{c}2.45 \\
(5.67)\end{array}$ & $\begin{array}{c}3.77 \\
(14.00)\end{array}$ & $\begin{array}{c}3.21 \\
(10.00)\end{array}$ & $\begin{array}{c}2.83 \\
(10.00)\end{array}$ & $\begin{array}{c}2.60 \\
(8.00)\end{array}$ & $\begin{array}{c}1.90 \\
(4.00)\end{array}$ & $\begin{array}{c}2.10 \\
(4.00)\end{array}$ \\
\hline $\begin{array}{l}\mathrm{T}_{6^{-}} 2,4-\mathrm{D} 0.5 \mathrm{lit}_{\mathrm{ha}}^{-1} \mathrm{fb} \\
\text { butachlor } 1 \mathrm{lit} \mathrm{ha}^{-1}\end{array}$ & $\begin{array}{c}3.13 \\
(9.33)\end{array}$ & $\begin{array}{c}2.90 \\
(8.00)\end{array}$ & $\begin{array}{c}3.50 \\
(12.00)\end{array}$ & $\begin{array}{c}3.15 \\
(9.50)\end{array}$ & $\begin{array}{c}4.90 \\
(24.00)\end{array}$ & $\begin{array}{c}3.67 \\
(13.00)\end{array}$ & $\begin{array}{c}4.23 \\
(18.00)\end{array}$ & $\begin{array}{c}4.00 \\
(15.60)\end{array}$ & $\begin{array}{c}2.83 \\
(8.00)\end{array}$ & $\begin{array}{c}2.70 \\
(6.80)\end{array}$ \\
\hline $\begin{array}{l}\mathrm{T}_{7}-\text { Metribuzin } 0.250 \\
\mathrm{Kg} \mathrm{ha}^{-1} \mathrm{fb} \text { butachlor } 1 \\
\text { lit ha }\end{array}$ & $\begin{array}{c}3.09 \\
(9.33)\end{array}$ & $\begin{array}{c}2.80 \\
(7.33)\end{array}$ & $\begin{array}{c}4.17 \\
(17.00)\end{array}$ & $\begin{array}{c}3.71 \\
(13.50)\end{array}$ & $\begin{array}{c}5.83 \\
(34.00)\end{array}$ & $\begin{array}{c}4.67 \\
(22.00)\end{array}$ & $\begin{array}{c}4.03 \\
(16.00)\end{array}$ & $\begin{array}{c}3.79 \\
(14.00)\end{array}$ & $\begin{array}{c}3.03 \\
(9.00)\end{array}$ & $\begin{array}{l}3.12 \\
(9.50)\end{array}$ \\
\hline $\begin{array}{l}\mathrm{T}_{8^{-}} 2,4-\mathrm{D} 0.5 \text { lit ha } \\
\text { hand weeding } 30 \mathrm{DAS}\end{array}$ & $\begin{array}{c}2.78 \\
(7.33)\end{array}$ & $\begin{array}{c}2.71 \\
(7.00)\end{array}$ & $\begin{array}{c}2.30 \\
(6.00)\end{array}$ & $\begin{array}{c}2.17 \\
(4.67)\end{array}$ & $\begin{array}{c}3.10 \\
(12.00)\end{array}$ & $\begin{array}{c}2.60 \\
(8.00)\end{array}$ & $\begin{array}{c}3.43 \\
(12.00)\end{array}$ & $\begin{array}{c}3.10 \\
(12.00)\end{array}$ & $\begin{array}{c}2.70 \\
(7.00)\end{array}$ & $\begin{array}{c}2.73 \\
(7.00)\end{array}$ \\
\hline $\begin{array}{l}\text { T9- Hand Weeding } 30 \\
\text { DAS }\end{array}$ & $\begin{array}{c}1.94 \\
(4.00)\end{array}$ & $\begin{array}{c}1.29 \\
(1.33)\end{array}$ & $\begin{array}{c}1.90 \\
(4.00)\end{array}$ & $\begin{array}{c}1.32 \\
(2.00)\end{array}$ & $\begin{array}{l}2.50 \\
(8.00)\end{array}$ & $\begin{array}{c}0.71 \\
(0.00)\end{array}$ & $\begin{array}{c}2.23 \\
(6.00)\end{array}$ & $\begin{array}{c}1.32 \\
(2.00)\end{array}$ & $\begin{array}{l}1.30 \\
(2.00)\end{array}$ & $\begin{array}{l}1.48 \\
(2.00)\end{array}$ \\
\hline $\mathrm{T}_{10^{-}}$Weedy check & $\begin{array}{c}4.37 \\
(18.67)\end{array}$ & $\begin{array}{c}4.81 \\
(22.67)\end{array}$ & $\begin{array}{c}5.37 \\
(29.00)\end{array}$ & $\begin{array}{c}5.13 \\
(26.00)\end{array}$ & $\begin{array}{c}7.63 \\
(58.00)\end{array}$ & $\begin{array}{c}6.51 \\
(42.00)\end{array}$ & $\begin{array}{c}7.13 \\
(50.00)\end{array}$ & $\begin{array}{c}7.09 \\
(50.00)\end{array}$ & $\begin{array}{c}5.07 \\
(26.00)\end{array}$ & $\begin{array}{c}5.50 \\
(30.00)\end{array}$ \\
\hline SEm \pm & 0.35 & 0.22 & 0.44 & 0.39 & 0.66 & 0.46 & 0.55 & 0.66 & 0.43 & 0.27 \\
\hline $\begin{array}{l}\text { Treatment (T) CD } \\
(\mathbf{P}=\mathbf{0 . 0 5})\end{array}$ & 1.01 & 0.65 & 1.29 & 1.14 & 1.94 & 1.36 & 1.61 & 1.91 & 1.25 & 0.79 \\
\hline
\end{tabular}

$*$ (Data subjected to square root $\mathrm{x}+0.5$ transformation and figures in parenthesis are original value) 
Table.5 Grain yield, straw yield and harvest index of wheat as influenced by different treatments under wheat- Eucalyptus tereticornis based agroforestry system

\begin{tabular}{|c|c|c|c|c|c|c|c|}
\hline \multirow{2}{*}{\multicolumn{2}{|c|}{ Treatment }} & \multicolumn{2}{|c|}{$\begin{array}{l}\text { Grain Yield } \\
\qquad\left(\mathrm{q} h \mathrm{a}^{-1}\right)\end{array}$} & \multicolumn{2}{|c|}{$\begin{array}{c}\text { Straw Yield } \\
\left(\mathbf{q} \mathrm{ha}^{-1}\right)\end{array}$} & \multicolumn{2}{|c|}{$\begin{array}{c}\text { Harvest Index } \\
(\%)\end{array}$} \\
\hline & & $\begin{array}{c}2016- \\
17\end{array}$ & $\begin{array}{c}2017- \\
18\end{array}$ & $\begin{array}{c}2016- \\
17\end{array}$ & $\begin{array}{l}2017- \\
18\end{array}$ & $\begin{array}{c}2016- \\
17\end{array}$ & 2017-18 \\
\hline $\mathbf{T}_{1}$ & 2, 4-D 0.5 lit $\mathrm{ha}^{-1}$ & 16.67 & 15.17 & 39.92 & 31.21 & 29.45 & 32.73 \\
\hline $\mathbf{T}_{2}$ & Metribuzin $0.250 \mathrm{Kg} \mathrm{ha}^{-1}$ & 17.04 & 15.32 & 40.10 & 31.85 & 29.83 & 32.48 \\
\hline $\mathbf{T}_{\mathbf{3}}$ & Butachlor 1 lit ha ${ }^{-1}$ & 13.97 & 12.85 & 35.41 & 28.94 & 28.27 & 30.79 \\
\hline $\mathbf{T}_{4}$ & $\begin{array}{l}\text { Clodinafop-propargyl } 0.140 \\
\mathrm{~kg} \mathrm{ha}^{-1}\end{array}$ & 17.63 & 16.18 & 41.36 & 34.52 & 29.89 & 31.89 \\
\hline $\mathbf{T}_{5}$ & $\begin{array}{l}2,4-\mathrm{D} 0.5 \text { lit ha }^{-1} \mathrm{fb} \\
\text { metribuzin } 0.250 \mathrm{Kg} \mathrm{ha}^{-1}\end{array}$ & 15.84 & 15.23 & 38.82 & 32.64 & 28.97 & 31.82 \\
\hline $\mathbf{T}_{6}$ & $\begin{array}{l}\text { 2, 4-D } 0.5 \text { lit ha }{ }^{-1} f b \text { butachlor } \\
1 \text { lit ha }\end{array}$ & 15.27 & 14.17 & 37.67 & 31.95 & 28.84 & 30.70 \\
\hline$\overline{\mathbf{T}_{7}}$ & $\begin{array}{l}\text { Metribuzin } 0.250 \mathrm{Kg} \mathrm{ha}^{-1} f b \\
\text { butachlor } 1 \text { lit ha }\end{array}$ & 15.00 & 13.70 & 36.50 & 27.99 & 29.14 & 32.84 \\
\hline $\mathbf{T}_{8}$ & $\begin{array}{l}\text { 2, 4-D } 0.5 \text { lit ha }^{-1}+\text { hand } \\
\text { weeding } 30 \text { DAS }\end{array}$ & 17.19 & 16.04 & 40.22 & 30.56 & 29.95 & 34.69 \\
\hline $\mathrm{T}_{9}$ & Hand Weeding 30 DAS & 19.75 & 18.20 & 46.54 & 39.72 & 29.80 & 31.41 \\
\hline $\mathbf{T}_{10}$ & Weedy check & 13.07 & 12.07 & 34.99 & 28.67 & 27.23 & 29.68 \\
\hline & SEm \pm & 0.40 & 0.54 & 0.82 & 1.37 & 0.71 & 0.91 \\
\hline Tre & tment $(\mathrm{T}) \mathrm{CD}(\mathrm{P}=0.05)$ & 1.17 & 1.57 & 2.39 & 3.99 & 2.08 & 2.64 \\
\hline Yea & $(\mathrm{Y}) \mathrm{CD}(\mathrm{P}=0.05)$ & - & - & - & - & - & - \\
\hline Inte & action (YxT) CD ( $\mathrm{P}=0.05)$ & - & - & - & - & - & - \\
\hline
\end{tabular}

\section{Rumex dentatus}

The perusal of data (Table 1) showed that hand weeding recorded lower weed density (T9: 0.67 and $0.00 \mathrm{~m}^{2}$ ) whereas weedy check recorded significantly higher weed density (T10: 7.33 and $7.00 \mathrm{~m}^{2}$ ). Among chemical weed control treatment 2, 4-D 0.5 lit ha ${ }^{-1}$ showed lower weed density (T1: 1.33 and $0.67 \mathrm{~m}^{2}$ ) followed by 2, 4-D 0.5 lit $^{\mathrm{ha}^{-1}+}$ hand weeding 30 DAS (T8: 2.00 and $1.33 \mathrm{~m}^{2}$ ) and 2, 4-D 0.5 lit ha $^{-1} \mathrm{fb}$ metribuzin $0.250 \mathrm{Kg}$ ha $^{-1}$ (T5: 1.67 and $1.60 \mathrm{~m}^{2}$ ) during both the year under wheat - Eucalyptus tereticornis based Agroforestry system.

The application of 2, 4-D 0.5 lit ha ${ }^{-1}$ (T1: 84.26 and $91.67 \%$ ), hand weeding 30 DAS $f b$
2, 4-D 0.5 lit ha $^{-1}$ (T8: 79.17 and 80.56), 2, 4D 0.5 lit ha $^{-1} \mathrm{fb}$ metribuzin $0.250 \mathrm{~kg} \mathrm{ha}^{-1}$ (T5: 74.54 and $76.59 \%$ ) were found higher weed control efficiency over weedy check $(0 \%)$, chlodinafop-propargyl $0.140 \mathrm{~kg} \mathrm{ha}^{-1}$ (T4: 38.42 and $50.00 \%$ ) and metribuzin $0.250 \mathrm{~kg}$ $\mathrm{ha}^{-1} \mathrm{fb}$ butachlor 1 lit ha ${ }^{-1}$ (T7: 39.81 and $49.01 \%$ ) during both the year (Table 2) Similar views have also been reported by Saini and Chopra (2015) and Singh et al., (2015).

Lowest weed dry weight of Rumex dentatus was found under hand weeding 30 DAS (T9: $8.00 \mathrm{~g} \mathrm{~m}^{-2}$ ) followed by 2, 4-D 0.5 lit ha $^{-1}$ (T1: $8.00 \mathrm{~g} \mathrm{~m}^{-2}$ ), hand weeding $30 \mathrm{DAS}+2,4 \mathrm{D}$ 0.5 lit ha $^{-1}$ (T8: $12.00 \mathrm{~g} \mathrm{~m}^{-2}$ ) and 2, 4-D 0.5 lit $\mathrm{ha}^{-1} \mathrm{fb}$ metribuzin $0.250 \mathrm{Kg} \mathrm{ha}^{-1}$ (T5: $14.00 \mathrm{~g}$ 
$\mathrm{m}^{-2}$ ) over Weedy check which found significantly higher (T10: $58.00 \mathrm{~g} \mathrm{~m}^{-2}$ ) Rumex dentatus dry weight. During second year 2017-18 hand weeding showed signicantly lower weeds dry weight over weedy check and all the management practices.

The application of 2,4-D @ $0.5 \mathrm{lha}^{-1}$ (T1: $4.00 \mathrm{~g} \mathrm{~m}^{-2}$ ) followed by 2, $4 \mathrm{D} 0.5 \mathrm{lit} \mathrm{ha}^{-1}+$ hand weeding 30 DAS (T8: $8.00 \mathrm{~g} \mathrm{~m}^{-2}$ ) were also reduced the dry weight of Rumex dentatus (Table 3). Similar finding also reported by Amare et al., (2014) and choudhry et al., (2016).

\section{Launaea nudicaulis}

The perusal of data (Table 1) showed that hand weeding recorded lower weed density (T9: 1.00 and $0.33 \mathrm{~m}^{2}$ ) whereas weedy check recorded significantly higher weed density (T10: 8.33 and $8.33 \mathrm{~m}^{2}$ ).

Among chemical weed control treatment 2, 4D 0.5 lit ha ${ }^{-1}$ showed lower weed density (T1: 1.67 and $1.00 \mathrm{~m}^{2}$ ) followed by 2, 4-D 0.5 lit $\mathrm{ha}^{-1}+$ hand weeding 30 DAS (T8: 2.00 and $2.00 \mathrm{~m}^{2}$ ) and 2, 4-D 0.5 lit ha $^{-1} \mathrm{fb}$ metribuzin $0.250 \mathrm{Kg} \mathrm{ha}^{-1}$ (T5: 1.67 and $1.33 \mathrm{~m}^{2}$ ) during both the year under wheat - Eucalyptus tereticornis based Agroforestry system.

The application of weed management practices the highest weed control efficiency was found under hand weeding 30 DAS (T9: 87.96 and $95.83 \%$ ) followed by 2, 4-D 0.5 lit $\mathrm{ha}^{-1}$ (T1: 79.63 and 86.90), 2, 4-D 0.5 lit ha $^{-1}$ $f b$ metribuzin $0.250 \mathrm{Kg} \mathrm{ha}^{-1}$ (T5: 80.09 and $82.14 \%$ ) and 2, 4-D 0.5 lit ha $^{-1}+$ hand weeding 30 DAS (T8: 75.46 and $75.71 \%$ ) over weedy check (T10: $0.00 \%$ ) during both the year under wheat - Eucalyptus tereticornis based Agroforestry system (Table 2). Similar views have also been reported by Saini and Chopra (2015) and Singh et al., (2015).
Among weed control practices the hand weeding had found lowest weed dry weight (T9: 6.00 and $2.00 \mathrm{~g} \mathrm{~m}^{-2}$ ) at par with 2, 4-D 0.5 lit ha ${ }^{-1}$ (T1: 10.00 and $6.00 \mathrm{~g} \mathrm{~m}^{-2}$ ), 2, 4-D 0.5 lit $^{-1} \mathrm{fb}$ metribuzin $0.250 \mathrm{~kg} \mathrm{ha}^{-1}$ (T5: 10.00 and $8.00 \mathrm{~g} \mathrm{~m}^{-2}$ ) and 2, 4-D 0.5 lit ha $^{-1}+$ hand weeding 30 DAS (T8:12.00 and $12.00 \mathrm{~g}$ $\mathrm{m}^{-2}$ ) over weedy check which found significantly higher Weed dry weight (T10: 50.00 and $50.00 \mathrm{gm}^{-2}$ ) during both the year under wheat - Eucalyptus tereticornis based Agroforestry system (Table 3). Similar finding also reported by Amare et al., (2014) and choudhry et al., (2016).

\section{Melilotus indicus}

The result showed that hand weeding recorded lower weed density (T9: 0.67 and $0.67 \mathrm{~m}^{2}$ ) whereas weedy check recorded significantly higher weed density (T10: 8.67 and $9.33 \mathrm{~m}^{2}$ ). Among chemical weed control treatment 2, 4-D 0.5 lit ha ${ }^{-1}$ showed lower weed density (T1: 2.00 and $1.33 \mathrm{~m}^{2}$ ) followed by 2, 4-D 0.5 lit ha $^{-1} f b$ metribuzin $0.250 \mathrm{Kg}$ $\mathrm{ha}^{-1}$ (T5: 1.33 and $1.33 \mathrm{~m}^{2}$ ) and 2, 4-D 0.5 lit $\mathrm{ha}^{-1}+$ hand weeding 30 DAS (T8: 2.33 and $2.33 \mathrm{~m}^{2}$ ) during both the year under wheat Eucalyptus tereticornis based Agroforestry system (Table 1).

The application of weed management practices the highest weed control efficiency was found under hand weeding 30 DAS (T9: 94.44 and $93.06 \%$ ) followed by 2, 4-D 0.5 lit ha $^{-1} f b$ metribuzin $0.250 \mathrm{Kg} \mathrm{ha}^{-1}$ (T5: 80.95 and $86.94 \%)$ and 2, 4-D 0.5 lit ha ${ }^{-1}$ (T1: 79.37 and $81.39 \%$ ) over Weedy check (T10: $0 \%$ ) (Table 2). Similar views have also been reported by, Saini and Chopra (2015) and Singh et al., (2015).

The lowest weed dry weight of Melilotus indicus was found under hand Weeding 30 DAS (T9: 2.00 and $2.00 \mathrm{~g} \mathrm{~m}^{-2}$ ) at par with 2, 4-D 0.5 lit ha $^{-1} f b$ metribuzin $0.250 \mathrm{Kg} \mathrm{ha}^{-1}$ 
(T5: 4.00 and $4.00 \mathrm{~g} \mathrm{~m}^{-2}$ ) and 2, 4-D 0.5 lit ha 1 (T1: 6.00 and $5.00 \mathrm{~g} \mathrm{~m}^{-2}$ ) which was significantly superior over weedy check (T10: 26.00 and $30.00 \mathrm{~g} \mathrm{~m}^{-2}$ ) during both the year (Table 3). Similar finding also reported by Pradhan and Chakraborti (2010), Tiwari et al., (2011) Pisal and Sagarka (2013), Amare et al., (2014) and choudhry et al., (2016).

\section{Grain yield}

The significantly higher grain yield was found under hand weeding 30 DAS (T9; 19.75 and $18.20 \mathrm{q} \mathrm{ha}^{-1}$ ) which was significantly superior over weedy check (T10: 13.07 and $12.02 \mathrm{q}$ $\mathrm{ha}^{-1}$ ) during both the year. Among herbicidal treatments the higher grain yield was found under chodinafop-propargyl $0.140 \mathrm{~kg} \mathrm{ha}^{-1}$ (T4: 17.63 and $16.18 \mathrm{q} \mathrm{ha}^{-1}$ ) followed by 2, 4D 0.5 lit $\mathrm{ha}^{-1}+$ hand weeding 30 DAS (T8: 17.19 and $16.04 \mathrm{q} \mathrm{ha}^{-1}$ ), metribuzin $0.250 \mathrm{Kg}$ $\mathrm{ha}^{-1}$ (T2: 17.04 and $15.32 \mathrm{q} \mathrm{ha}^{-1}$ ) and 2, 4-D 0.5 lit $\mathrm{ha}^{-1}$ (16.67 and $15.32 \mathrm{q} \mathrm{ha}^{-1}$ ) over control (T10: 13.07 and 12.07 q ha-1), followed by butachlore 1 lit ha ${ }^{-1}$ (13.97 and $12.85 \mathrm{q} \mathrm{ha}^{-1}$ ) during both the year. The $33.82 \%$ and $33.68 \%$ yield reduction was found under weedy check treatments over hand weeding 30 DAS during both the year under wheat - Eucalyptus tereticornis based Agroforestry system (Table 4). The findings are in close conformity to the findings of Brar et al., (2002), Yadav et al., (2009) and Chander et al., (2014).

\section{Straw yield}

The significantly higher straw yield was found under hand weeding 30 DAS (T9; 46.54 and $39.72 \mathrm{q} \mathrm{ha}^{-1}$ ) which was significantly superior over weedy check (T10: 34.99 and $28.67 \mathrm{q} \mathrm{ha}^{-1}$ ) and rest of the weed control treatments. Among herbicidal treatment the higher straw yield was found under chodinafop-propargyl $0.140 \mathrm{~kg} \mathrm{ha}^{-1}$ (T4: 41.36 and $34.52 \mathrm{q} \mathrm{ha}^{-1}$ ) over weedy check (T10: 34.99 and 28.67 $\mathrm{q} \mathrm{ha}^{-1}$ ), followed by butachlor 1 lit ha ${ }^{-1}$ (T3: 35.41 and $28.94 \mathrm{q}$ $\mathrm{ha}^{-1}$ ). The $24.81 \%$ and $11.05 \%$ straw yield reduction was found under weedy check over hand weeding 30 DAS during both the year (Table 4).

\section{Harvest index}

The higher harvest index was found under 2, 4-D 0.5 lit ha $^{-1}+$ Hand weeding 30 DAS (T8: $29.95 \%$ ) over weedy check (T10: $27.23 \%$ ) during first year (2016-17) and During second year (2017-18), higher straw yield was found under 2, 4-D 0.5 lit ha ${ }^{-1}+$ hand weeding 30 DAS (T8: 34.69\%) followed by metribuzin $0.250 \mathrm{Kg} \mathrm{ha}^{-1} \mathrm{fb}$ butachlor 1 lit ha ${ }^{-1}$ (T7: $32.84 \%$ ), 2, 4-D 0.5 lit ha $^{-1}$ (T1: 32.73\%) and metribuzin $0.250 \mathrm{Kg} \mathrm{ha}^{-1}$ (T2: $32.48 \%$ ) over weedy check (T10: 29.68\%) (Table 5).

From the two year experiment result concluded that, the hand weeding was superior to control all type of weed under wheat - Eucalyptus tereticornis based Agroforestry system. Among the weed management practices concluded that clodinafop-propagyl at $0.140 \mathrm{~kg} \mathrm{ha}^{-1}$ reduce Phalaris minor and 2, 4-D 0.5 lit ha $^{-1}$ reduce weed density of broad leaved weed whereas, 2,4D+ hand weeding $30 \mathrm{DAS}$ and, 4-D 0.5 lit $\mathrm{ha}^{-1} \mathrm{fb}$ metribuzin $0.250 \mathrm{Kg} \mathrm{ha}^{-1}$ reduce weed density and dry weight of both broad leaved and narrow leaf weed at all stage of crop growth. These treatments also increase grain yield and straw yield over weedy check plot under wheat - Eucalyptus tereticornis based Agroforestry system.

\section{References}

Amare T, Sharma JJ and Zewdie K. 2014. Effect of Weed Control Methods on Weeds and Wheat (Triticum aestivum L.) Yield. World Journal of Agricultural Research, Vol. 2, No. 3, 124-128. 
Angiras NN, Kumar S, Rana SS and Sharma N. 2008.Standardization of dose and time of application of clodinafoppropargyl to manage weeds in wheat. Himachal Journal of Agricultural Research 34(2): 15-18.

Brar, LS, Waiia, U.S., and Dhaliwal (2002). Performance of Clodinafop-p-propargyi for the control of grassy weeds in wheat Journal of Res., PAL). Ludhyana. 36, 3 and 4): 187-190.

Chander N, Kumar S, Rana SS and Ramesh. 2014. Weed competition, yield attributes and yield in soybean (Glycine max)-wheat (Triticum aestivum) cropping system as affected by herbicides. Indian Journal of Agronomy 59 (3): 377-384.

Choudhary D, Singh PK, Chopra NK and Rana SC. 2016. Effect of herbicides and herbicide mixtures on weeds in wheat. Indian J. Agric. Res., 50 (2): 107-112.

Dixit A and Singh VP. 2008. Efficacy of a ready mix application of carfentrazone plus isoproturon (affinity) to control weed in wheat (Triticum aestivum). Indian Journal of Agricultural Sciences 78(6): 495-97.

Gill GS and Kumar V. 1969. 'Weed index' a new method for reporting weed control trails. Indian Journal of Agronomy 14(1): pp96-97.

Gomez AK and Gomez AA.1984.Statistical procedure for agricultural research, II Edition, A Willey- International Science Publication, John Wiley and Sons. New Delhi, India. 680p.

Kumar N, Mina BL, Singh KP, Chandra S, Kumar M and Shrivastawa AK. 2010. Weed control for yield maximization in CLI wheat (Triticum aestivum L.) in Indian Himalayas. Indian Journal of Agronomy. 55 (2): 119-122.

Kumar S, Angiras NN and Rana SS. 2011. Bio-efficacy of clodinafop-propargyl + metsulfuron methyl against complex weed flora in wheat. Indian Journal of Weed Science 43(3\&4):195 198.

Mani VS, Malla ML, Gautam KC and Bhagwandas. 1973. Weed killing chemicals in Potato cultivation. Indian Farming 27(8): pp17-18.

Nichiporovich AA. 1967. Photosynthesis of productive system. Jerusalem: Israel Programme Science Tansi, 182.

Nural-Islam MD and Johanson HB. 1987. Physical chemical tests -a basis of selecting the size of wheat flour. J. Food Sci. Technol., 24: 136-145.

Pisal RR and Sagarka BK. 2013.Integrated weed management in wheat with new molecules. Indian Journal of Weed Science 45(1): 25-28.

Pradhan AC and Chakraborti P. 2010. Quality wheat seed production through integrated weed management. Indian J. Weed Sci. 42 (3 \& 4): 159-162

Puri S and Bangarwa KS. 1992. Effects of trees on the yield of irrigated wheat crop in semi-arid regions. Agrofores. Syst., 20: 229-241.

Saini MK and Chopra S. 2015. Influence of weed control methods on weeds, yield, energetics and economics of basmati rice (Oryza sativa) under submountaineous conditions of Punjab Indian Journal of Agronomy 60 (3): 410-413.

Singh R, Singh AP, Chaturvedi S, Rekha, Pal $\mathrm{R}$ and Pal J. 2015. Metribuzin + clodinafop-propargyl effects on complex weed flora in wheat and its residual effect on succeeding crop Indian Journal of Weed Science 47(4): 362-365.

Singh, S., Sharma, S.D., Punia, S.S. and Singh, H. 2005. Performance of tank mixture of Metribuzin with Clodinafopp-propargyl and Fenxaprop of the control of mixed weed flora in wheat. Indian Journal of Weed Science, 37(1 and 2): 9-12. 
Tiwari RK, Khan IM, Singh N and Jha A. 2011.Chemical weed control in wheat through on farm demonstrations in Rewa district of Madhya Pradesh. Indian J. Weed Sci. 43 (3 \& 4): 215216.

Verma P, Bijalwan A, Manmohan, Dobriyal JR, Swamy SL and Thakur TK. 2017. A paradigm shift in agroforestry practices in Uttar Pradesh. Current Science, VOL. 112, NO. 3, 509-516.

Yadav, D. B., Punia, S.S., Yadav, A., Singh, S. and Lai, R. (2009). Pinoxaden, an alternate herbicide against little seed canary grass (Phalaris minor) in wheat. Indian Journal of Agronomy, 54(4): 433-437.

\section{How to cite this article:}

Atul Singh, K.K. Jain and Upadhyaya, S.D. 2018. Effect of Weed Control Methods on Weeds and Wheat under Eucalyptus tereticornis Based Agroforestry System. Int.J.Curr.Microbiol.App.Sci. 7(08): 2856-2867. doi: https://doi.org/10.20546/ijcmas.2018.708.301 